\title{
A restriction estimate for a class of oscillatory integral operators along paraboloid
}

Shaozhen $\mathrm{Xu}^{1,3}$ and Wenjuan $\mathrm{Li}^{\mathrm{i}^{*}}$

"Correspondence:
liwj@nwpu.edu.cn
${ }^{2}$ School of Natural and Applied
Sciences, Northwestern
Polytechnical University, Xi'an, China
Full list of author information is
available at the end of the article

available at the end of the article

\section{Abstract}

In this paper we establish a restriction estimate for a class of oscillatory integral operators along a paraboloid,

$$
\mathbb{P}^{d-1}:=\left\{\left(x_{1}, \ldots, x_{d}\right): x_{d}=x_{1}^{2}+\cdots+x_{d-1}^{2}\right\} .
$$

Specifically, we consider the oscillatory integral operators defined by

$$
T_{m, n} f(x)=\int_{\mathbb{R}^{d}} e^{i\left(x_{1}^{m} \xi_{1}^{n}+\cdots+x_{d}^{m} \xi_{d}^{n}\right)} f(\xi) d \xi
$$

where $n, m$ are integers satisfying $2 \leq d<n \leq 2 m d$, then

$$
\left\|T_{m, n} f\right\|_{L^{2}\left(d \sigma, \mathbb{P}^{d-1} \cap B^{d}(0,1)\right)} \leq C_{m, n, d}\|f\|_{L^{p}\left(\mathbb{R}^{d}\right)}
$$

holds for $1<p \leq \frac{4 m d}{4 m d-n}$. A necessary condition is also given to ensure this boundedness.

MSC: $42 \mathrm{~B} 20 ; 47 \mathrm{G} 10$

Keywords: Restriction estimate; Oscillatory integral operator; Multidimensional Van der Corput; Knapp's counterexample

\section{Introduction}

The Fourier restriction conjecture has attracted a lot of attention in the development of modern harmonic analysis. It was raised by Stein in the 1960s and can be stated as follows (in its dual form).

Conjecture 1 Suppose $S_{0}$ is a compact subset of a smooth $(d-1)$-dimensional manifold $S$ with nonvanishing Guassian curvature and $d \sigma$ is the surface measure on $S$ induced by the Lebesgue measure on $\mathbb{R}^{n}$, then

$$
\int_{S_{0}}|\hat{f}(\xi)| d \sigma \leq C\|f\|_{L^{p}\left(\mathbb{R}^{d}\right)}
$$

holds for $1<p<\frac{2 d}{d+1}$. 
A natural idea to attack this problem is to establish a stronger estimate, for example the $L^{2}$ estimate, of the left hand side of (2). Therefore, the Hölder inequality and the finite measure of $S_{0}$ yield the corresponding inequality. Combining the $T T^{*}$ method with the Fourier decay of surface measure $d \sigma$, one may get the nontrivial estimate

$$
\|\hat{f}\|_{L^{2}(S, d \sigma)} \leq C\|f\|_{L^{p}\left(\mathbb{R}^{d}\right)}
$$

for

$$
1<p \leq \frac{4 d}{3 d+1} .
$$

It should be noted that our paper replaces the Fourier decay of $d \sigma$ by an oscillatory integral decay estimate. In [1], Tomas improved the estimate (4) to

$$
1<p<\frac{2 d+2}{d+3}
$$

up to the endpoint

$$
p=\frac{2 d+2}{d+3}
$$

The endpoint case was due to Stein. The inequality (3) for

$$
1<p \leq \frac{2 n+2}{n+3}
$$

is now known as the Tomas-Stein estimate; it is also sharp in view of Knapp's counterexample; see [2]. Now, we return to the conjecture 1. Obviously, the Tomas-Stein estimate gives rise to a highly nontrivial improvement on this conjecture. However, the case

$$
\frac{2 d+2}{d+3}<p<\frac{2 d}{d+1}
$$

was still unknown until the breakthrough of Bourgain [3] in 1991. Bourgain first improved the exponent in (5) by connecting the Fourier restriction with the behavior of Kakeya maximal operators. Later, a bilinear approach was developed by Wolff, Tao and other mathematicians, which refers to the interaction between two different surfaces. Since a bilinear restriction implies a linear restriction in some sense, improvements on a bilinear restriction naturally lead to improvements on linear restriction; this work can be found in [4-8]. In the first decade of the 21st century, the concentration on Fourier restriction turns to multilinear case. Some remarkable results were obtained in [9] by Bennett, Carbery and Tao; they proved the near-optimal multilinear Kakeya and restriction conjectures. But to what extent multilinear restriction can imply linear restriction was not clear until the breakthrough by Bourgain and Guth [10]. Later, Guth applied the polynomial partitioning method to the restriction problem and obtained the range for $p$,

$$
1<p<\frac{13}{9},
$$

in the 3-dimensional case; see [11]. 
The purpose of our paper is to extend the restriction estimate from Fourier transform to oscillatory integral operators. Xu and Yan have established some corresponding results on the $(d-1)$-dimensional unit sphere in [12]. The definition of the oscillatory integral operators is given by

$$
T_{\lambda}(f)(\xi)=\int_{\mathbb{R}^{d-1}} e^{i \lambda \phi(x, \xi)} \psi(x, \xi) f(x) d x
$$

where $\phi(x, \xi)$ is a smooth function and $\psi(x, \xi)$ is a cut-off function. For this kind of operator, we show the decay estimate

$$
\left\|T_{\lambda}\right\|_{L^{p} \rightarrow L^{q}} \leq C \lambda^{-\theta}
$$

for suitable $p, q, \theta$. Since the Fourier restriction operator is a Hörmander-type oscillatory integral operator, if letting $\lambda \rightarrow+\infty$ and some limiting arguments, the decay estimate will imply the restriction conjecture. Details can be found in [2]. The Hörmander-type oscillatory integral operator requires that the mixed Hessian matrix of phase function $\phi$ should have maximal rank $d-1$ on the support of $\psi$. Consider the dual operator of (1)

$$
T_{m, n}^{*} g(\xi)=\int_{\mathbb{R}^{d-1}} e^{i\left[x_{1}^{m} \xi_{1}^{n}+\cdots+\left(x_{1}^{2}+\cdots+x_{d-1}^{2}\right)^{m} \xi_{d}^{n}\right]} \tilde{\psi}(x) g(x) d x_{1} \cdots d x_{d-1} .
$$

It is obvious that the mixed Hessian matrix

$$
\left[\frac{\partial^{2} \phi}{\partial x_{j} \partial \xi_{k}}\right](x, \xi)=m n\left(\begin{array}{cccc}
x_{1}^{m-1} \xi_{1}^{n-1} & \cdots & 0 & 2 x_{1}|x|^{2(m-1)} \xi_{d}^{n-1} \\
\vdots & & & \\
0 & \ddots & 0 & \vdots \\
\vdots & & & \\
0 & \cdots & x_{d-1}^{m-1} \xi_{d-1}^{n-1} & 2 x_{d-1}|x|^{2(m-1)} \xi_{d}^{n-1}
\end{array}\right)
$$

has rank zero at $(x, \xi)=(0,0)$ since $n>2$. Therefore, (1) is not of Hörmander-type. For such non-Hörmander-type oscillatory integral operators we obtain the following restriction estimate.

Theorem 1 Suppose that $n, m, d$ are integers satisfying $2 \leq d<n \leq 2 m d$. Define the oscillatory integral operators $T_{m, n}$ as (1). Then, for all $1<p \leq \frac{4 m d}{4 m d-n}$,

$$
\left\|T_{m, n} f\right\|_{L^{2}\left(d \sigma, \mathbb{P}^{d-1} \cap B^{d}(0,1)\right)} \leq C_{m, n, d}\|f\|_{L^{p}\left(\mathbb{R}^{d}\right)},
$$

where d $\sigma$ is the surface measure on paraboloid and $B^{d}(0,1)$ is the unit ball in $n$-dimensional Euclidean space.

\section{Proof of Theorem 1}

Proof $T T^{*}$ transforms our boundedness estimate to scalar oscillatory integral estimate. For any Schwartz function $f$, we now compute the $L^{2}$ norm of $T_{m, n}(f)$ on the compact 
subset of $(n-1)$-dimensional paraboloid. It follows that

$$
\begin{aligned}
& \left\|T_{m, n}(f)\right\|_{L^{2}\left(\mathbb{P}^{d-1} \cap B^{d}(0,1)\right)}^{2} \\
& \quad=\left\langle T_{m, n}(f), T_{m, n}(f)\right\rangle_{\mathbb{P}^{d-1} \cap B^{d}(0,1)} \\
& \quad=\int_{\mathbb{P}^{d-1} \cap B^{d}(0,1)} T_{m, n}(f)(x) \overline{T_{m, n}(f)(x)} d \sigma(x) \\
& =\int_{\mathbb{R}^{d}} \int_{\mathbb{R}^{d}} \int_{\mathbb{P}^{d-1} \cap B^{d}(0,1)} e^{i\left[x_{1}^{m}\left(\xi_{1}^{n}-\eta_{1}^{n}\right)+\cdots+x_{d}^{m}\left(\xi_{d}^{n}-\eta_{d}^{n}\right)\right]} d \sigma(x) f(\xi) \overline{f(\eta)} d \xi d \eta \\
& \leq \int_{\mathbb{R}^{d}} \int_{\mathbb{R}^{d}}\left|\int_{\mathbb{P}^{d-1} \cap B^{d}(0,1)} e^{i\left[\left(x_{1}^{m}\left(\xi_{1}^{n}-\eta_{1}^{n}\right)+\cdots+x_{d}^{m}\left(\xi_{d}^{n}-\eta_{d}^{n}\right)\right]\right.} d \sigma(x)\right| f(\xi) \overline{f(\eta)} \mid d \xi d \eta .
\end{aligned}
$$

Denoting the inner integral in the last inequality by $K(\xi, \eta)$, then

$$
\left\|T_{m, n}(f)\right\|_{L^{2}\left(\mathbb{P}^{d-1} \cap B^{d}(0,1)\right)}^{2} \leq \int_{\mathbb{R}^{d}} \int_{\mathbb{R}^{d}}|K(\xi, \eta)||f(\xi) f(\eta)| d \xi d \eta .
$$

Set

$$
I(\lambda)=\int_{\mathbb{P} d-1 \cap B^{d}(0,1)} e^{i\left(\lambda_{1} x_{1}^{m}+\cdots+\lambda_{d} x_{d}^{m}\right)} d \sigma(x),
$$

where $\lambda=\left(\lambda_{1}, \ldots, \lambda_{d}\right)$. We desire to establish the decay estimate of $|I(\lambda)|$ with respect to $|\lambda| \gg 1$. In fact,

$$
\begin{aligned}
I(\lambda) & =\int_{\mathbb{P}^{d-1} \cap B^{d}(0,1)} e^{i\left(\lambda_{1} x_{1}^{m}+\cdots+\lambda_{d} x_{d}^{m}\right)} d \sigma(x) \\
& =\int_{U} e^{i\left(\lambda_{1} x_{1}^{m}+\cdots+\lambda_{d}\left(x_{1}^{2}+\cdots+x_{d-1}^{2}\right)^{m}\right)} \varphi\left(x_{1}, \ldots, x_{d-1}\right) d x_{1} \cdots d x_{d-1},
\end{aligned}
$$

where $U \subset \mathbb{R}^{d-1}$ is the projection of $\mathbb{P}^{d-1} \cap B^{d}(0,1)$ on $(d-1)$-dimensional Euclidean space and

$$
\varphi\left(x_{1}, \ldots, x_{d-1}\right)=\sqrt{1+4\left(x_{1}^{2}+\cdots+x_{d-1}^{2}\right)} .
$$

Note that the phase function of $I(\lambda)$ is a polynomial of degree $2 m$ with variable coefficients $\lambda_{1}, \ldots, \lambda_{d-1}$. To establish the decay estimate of $I(\lambda)$ with respect to $|\lambda|$, we should precisely describe the relation between decay estimate and these variable coefficients. The following lemma is exactly what we want.

Lemma 1 Let $P(x)=\sum_{|\alpha| \leq D} c_{\alpha} x^{\alpha}$ be a polynomial of degree $\leq D, \varphi$ be a smooth function in the unit ball $B^{d}(0,1)$, and $\Omega$ be any convex subset of the unit ball. Then

$$
\left|\int_{\Omega} e^{i P(x)} \varphi(x) d x\right| \leq C_{D, d}\left(\sum_{0<|\alpha| \leq D}\left|c_{\alpha}\right|\right)^{-\frac{1}{D}} \sup _{x \in B^{d}(0,1)}(|\varphi(x)|+|\nabla \varphi(x)|) .
$$

This uniform multidimensional Van der Corput lemma can be found in [13] and [14]. By applying this lemma to $I(\lambda)$, we can see that

$$
|I(\lambda)| \leq C_{m, d}\left(\left|\lambda_{1}\right|+\cdots+c_{m}\left|\lambda_{d}\right|\right)^{-\frac{1}{2 m}},
$$


where $c_{m}>1$ is a positive constant only depending on $m$. A pigeonholing argument yields

$$
|I(\lambda)| \leq C_{m, d}|\lambda|^{-\frac{1}{2 m}}
$$

Returning to our original argument, the decay estimate of $|I(\lambda)|$ naturally gives rise to an upper bound of $|K(\xi, \eta)|$,

$$
\begin{aligned}
|K(\xi, \eta)| & \leq C_{m, d}\left(\left|\xi_{1}^{n}-\eta_{1}^{n}\right|+\cdots+\left|\xi_{d}^{n}-\eta_{d}^{n}\right|\right)^{-\frac{1}{2 m}} \\
& \leq\left. C_{m, d}|| \xi_{1}\right|^{n}-\left|\eta_{1}\right|^{n}+\cdots+|\xi|_{d}^{n}-\left.\left|\eta_{d}\right|^{n}\right|^{-\frac{1}{2 m}} .
\end{aligned}
$$

Define a norm by

$$
|\xi|_{n}=\left(\left|\xi_{1}\right|^{n}+\cdots+\left|\xi_{d}\right|^{n}\right)^{\frac{1}{n}}
$$

Thus

$$
|K(\xi, \eta)| \leq\left. C_{m, d}|| \xi\right|_{n} ^{n}-\left.|\eta|_{n}^{n}\right|^{-\frac{1}{2 m}}
$$

Consequently,

$$
\begin{aligned}
& \left\|T_{m, n}(f)\right\|_{L^{2}\left(\mathbb{P}^{d-1} \cap B^{d}(0,1)\right)}^{2} \\
& \quad \leq\left. C_{m, d} \int_{\mathbb{R}^{d}} \int_{\mathbb{R}^{d}}|| \xi\right|_{n} ^{n}-\left.|\eta|_{n}^{n}\right|^{-\frac{1}{2 m}}|f(\xi) f(\eta)| d \xi d \eta .
\end{aligned}
$$

For positive $a, b$, define a fractional integration operator by

$$
R_{a}^{b}(f)(\eta)=\left.\int_{\mathbb{R}^{d}}|| \xi\right|_{a} ^{a}-\left.|\eta|_{a}^{a}\right|^{-\frac{d}{b}} f(\xi) d \xi
$$

The boundedness of $R_{a}^{b}$ on Lebesgue spaces was essentially established in [15]; it states the following.

Lemma 2 For $n<a \leq b$,

1. $R_{a}^{b}$ is of weak type $(1, b / a)$.

2. $R_{a}^{b}$ is bounded from $L^{p}\left(\mathbb{R}^{d}\right)$ to $L^{q}\left(\mathbb{R}^{d}\right)$, whenever $\frac{1}{p}=\frac{1}{q}+\frac{b-a}{b}$ and $1<p<\frac{b}{b-a}$.

Obviously,

$$
\left\|T_{m, n}(f)\right\|_{L^{2}\left(\mathbb{P}^{d-1} \cap B^{d}(0,1)\right)}^{2} \leq C_{m, d} \int_{\mathbb{R}^{d}} R_{n}^{2 m d}(|f|)(\xi)|f(\eta)| d \eta .
$$

The Hölder inequality implies

$$
\left\|T_{m, n}(f)\right\|_{L^{2}\left(\mathbb{P}^{d-1} \cap B^{d}(0,1)\right)}^{2} \leq C_{m, d}\left\|R_{n}^{2 m d}(|f|)\right\|_{L^{p^{\prime}}}\|f\|_{L^{p}} .
$$

We use (2) and set

$$
\frac{1}{p}=\frac{1}{p^{\prime}}+\frac{2 m d-n}{2 m d} .
$$


Simple computation gives

$$
p=\frac{4 m d}{4 m d-n} .
$$

By the density of Schwartz functions in $L^{p}$, a limiting argument yields the same restriction phenomena on $L^{p}$. The proof of Theorem 1 is completed.

Remark 1 In fact, as Xu and Yan have done in [12], Theorem 1 can be extended to oscillatory integral operators with more general phase functions.

\section{The necessary condition to guarantee (7)}

In this part, we will construct an example in the spirit of Knapp's counterexample to show the necessary condition of (7). Then we have following.

Theorem 2 If (7) holds, the exponent p must obey

$$
p \leq \frac{2 m(d+1)}{2 m(d+1)-(d-1) n} .
$$

Proof If the inequality

$$
\left[\int_{\mathbb{P}^{d-1} \cap B^{d}(0,1)}\left|T_{m, n} f(x)\right|^{2} d \sigma\right]^{\frac{1}{2}} \leq C_{m, n, d}\|f\|_{L^{p}\left(\mathbb{R}^{d}\right)}
$$

holds, then, for sufficiently small $\delta>0$, we have

$$
\begin{aligned}
{\left[\int_{R_{\delta} \cap \mathbb{P}^{d-1} \cap B^{d}(0,1)}\left|T_{m, n} f(x)\right|^{2} d \sigma\right]^{\frac{1}{2}} } & \leq\left[\int_{\mathbb{P}^{d-1} \cap B^{d}(0,1)}\left|T_{m, n} f(x)\right|^{2} d \sigma\right]^{\frac{1}{2}} \\
& \leq C_{m, n, d}\|f\|_{L^{p}\left(\mathbb{R}^{d}\right)},
\end{aligned}
$$

where

$$
R_{\delta}=\left\{\left(x_{1}, \ldots, x_{d}\right):\left|x_{j}\right| \leq c \delta^{\frac{1}{2}}, x_{d} \leq c \delta, j=1, \ldots, d-1\right\},
$$

where $0<c<1$ is a small absolute constant determined later. Let

$$
f(\xi)=\prod_{k=1}^{d} \chi_{I_{k}}\left(\xi_{k}\right)
$$

where $I_{d}=\left[-c^{\prime} \delta^{-\frac{m}{n}}, c^{\prime} \delta^{-\frac{m}{n}}\right]$ and $I_{k}=\left[-c^{\prime} \delta^{-\frac{m}{2 n}}, c^{\prime} \delta^{-\frac{m}{2 n}}\right](1 \leq k \leq d-1)$ are intervals and $0<$ $c^{\prime}<1$ is a sufficiently small constant, determined later. For a fixed point $x \in R_{\delta} \cap \mathbb{P}^{d-1} \cap$ $B^{d}(0,1)$, we have

$$
\begin{aligned}
& \left|T_{m, n} f(x)\right| \\
& \quad=\left|\int_{\mathbb{R}^{d}} e^{i\left(x_{1}^{m} \xi_{1}^{n}+\cdots+x_{d}^{m} \xi_{d}^{n}\right)} \prod_{k=1}^{d} \chi_{I_{k}}\left(\xi_{k}\right) d \xi_{1} \cdots d \xi_{d}\right|
\end{aligned}
$$




$$
\begin{aligned}
& =\prod_{k=1}^{d}\left|\int_{\mathbb{R}} e^{i x_{k}^{m} \xi_{k}^{n}} \chi_{I_{k}}\left(\xi_{k}\right) d \xi_{k}\right| \\
& :=\prod_{k=1}^{d}\left|F_{k}\left(x_{k}\right)\right| .
\end{aligned}
$$

Now, we give the lower bound of $F_{k}(x)$. In fact, for $1 \leq k \leq d-1$, we have

$$
\begin{aligned}
& \left|F_{k}\left(x_{k}\right)\right| \\
& \quad=\left|\int_{-\infty}^{+\infty} e^{i x_{k}^{m} \xi_{k}^{n}} \chi_{I_{k}}\left(\xi_{k}\right) d \xi_{k}\right| \\
& \quad=\left|\int_{-c^{\prime} \delta^{-\frac{m}{2 n}}}^{c^{\prime} \delta^{-\frac{m}{2 n}}} e^{i x_{k}^{m} \xi_{k}^{n}} d \xi_{k}\right| \\
& =\left|\int_{-c^{\prime} \delta^{-\frac{m}{2 n}}}^{c^{\prime} \delta^{-\frac{m}{2 n}}}\left[\cos \left(x_{k}^{m} \xi_{k}^{n}\right)+i \sin \left(x_{k}^{m} \xi_{k}^{n}\right)\right] d \xi_{k}\right| \\
& \quad \geq\left|\int_{-c^{\prime} \delta^{-\frac{m}{2 n}}}^{c^{\prime} \delta^{-\frac{m}{2 n}}} \cos \left(x_{k}^{m} \xi_{k}^{n}\right) d \xi_{k}\right| .
\end{aligned}
$$

Since $x \in R_{\delta} \cap \mathbb{P}^{d-1} \cap B^{d}(0,1)$, we have

$$
\left|F_{k}\left(x_{k}\right)\right| \geq c^{\prime} \delta^{-\frac{m}{2 n}}
$$

if

$$
c^{m}\left(c^{\prime}\right)^{n}<2^{-10}
$$

With the same assumptions on $c$ and $c^{\prime}$, we also have $\left|F_{d}\left(x_{d}\right)\right| \geq c^{\prime} \delta^{-\frac{m}{n}}$.

By plugging the constructed function (11) into the inequality (10), we obtain

$$
\begin{aligned}
C_{m, n, d}\|f\|_{L^{p}\left(\mathbb{R}^{d}\right)} & =C_{m, n, d}\left(2 c^{\prime}\right)^{\frac{d}{p}} \delta^{-\frac{m(d+1)}{2 n p}} \\
& \geq\left[\int_{R_{\delta} \cap \mathbb{P}^{d-1} \cap B^{d}(0,1)}\left|T_{m, n} f(x)\right|^{2} d \sigma\right]^{\frac{1}{2}} \\
& =\left[\int_{R_{\delta} \cap \mathbb{P}^{d-1} \cap B^{d}(0,1)}\left|\prod_{k=1}^{d}\right| F_{k}\left(x_{k}\right)||^{2} d \sigma\right]^{\frac{1}{2}} \\
& \gtrsim\left[\sigma\left(R_{\delta} \cap \mathbb{P}^{d-1} \cap B^{d}(0,1)\right)\right]^{\frac{1}{2}} \delta^{-\frac{m(d+1)}{2 n}} .
\end{aligned}
$$

From (8), the Jacobi of $d \sigma$ can be bounded from below and above on the unit ball. Thus

$$
\sigma\left(R_{\delta} \cap \mathbb{P}^{d-1} \cap B^{d}(0,1)\right) \approx \delta^{\frac{d-1}{2}} .
$$

It means that

$$
1 \gtrsim \delta^{\frac{m(d+1)}{2 n p}-\frac{m(d+1)}{2 n}+\frac{d-1}{4}}
$$


holds for all sufficiently small $\delta$. This requires

$$
\frac{m(d+1)}{2 n p}-\frac{m(d+1)}{2 n}+\frac{d-1}{4} \geq 0,
$$

which equals

$$
p \leq \frac{2 m(d+1)}{2 m(d+1)-(d-1) n} .
$$

We finish our proof of necessity.

\section{Remark 2 It is easy to verify that}

$$
\frac{4 m d}{4 m d-n}<\frac{2 m(d+1)}{2(d+1) m-(d-1) n} .
$$

Our result is not sharp since there is a gap between sufficient and necessary conditions.

\section{Funding}

The second author is supported by National Natural Science Foundation of China (No. 11601427), The Fundamental Research Funds for the Central Universities (No. 3102017zy035).

\section{Competing interests}

The authors declare that they have no competing interests.

\section{Authors' contributions}

All authors worked in coordination and contributed equally. All authors carried out the proof, read and approved the final version of the manuscript.

\section{Author details}

${ }^{1}$ School of Mathematical Sciences, University of Chinese Academy of Sciences, Beijing, China. ${ }^{2}$ School of Natural and Applied Sciences, Northwestern Polytechnical University, Xi'an, China. ${ }^{3}$ Department of Mathematics, University of Illinois at Urbana-Champaign, Urbana, USA.

\section{Publisher's Note}

Springer Nature remains neutral with regard to jurisdictional claims in published maps and institutional affiliations.

Received: 12 June 2018 Accepted: 10 January 2019 Published online: 23 January 2019

\section{References}

1. Tomas, P.A.: A restriction theorem for the Fourier transform. Bull. Am. Math. Soc. 81(2), 477-478 (1975)

2. Stein, E.M., Murphy, T.S.: Harmonic Analysis: Real-Variable Methods, Orthogonality, and Oscillatory Integrals. Princeton University Press, New Jersey (1993)

3. Bourgain, J.: Besicovitch type maximal operators and applications to Fourier analysis. Geom. Funct. Anal. 1(2), 147-187 (1991)

4. Wolff, T.H.: An improved bound for Kakeya type maximal functions. Rev. Mat. Iberoam. 11(3), 651-674 (1995)

5. Tao, T., Vargas, A., Vega, L.: A bilinear approach to the restriction and kakeya conjectures. J. Am. Math. Soc. 11(4), 967-1000 (1998)

6. Tao, T., Vargas, A.: A bilinear approach to cone multipliers i. Restriction estimates. Geom. Funct. Anal. 10(1), 185-215 (2000)

7. Wolff, T.: A sharp bilinear cone restriction estimate. Ann. Math. (2) 153(3), 661-698 (2001)

8. Tao, T.: A sharp bilinear restriction estimate for paraboloids. Geom. Funct. Anal. 13(6), 1359-1384 (2003)

9. Bennett, J., Carbery, A., Tao, T.: On the multilinear restriction and Kakeya conjectures. Acta Math. 196(2), 261-302 (2006)

10. Bourgain, J., Guth, L.: Bounds on oscillatory integral operators based on multilinear estimates. Geom. Funct. Anal. 21(6), 1239-1295 (2011)

11. Guth, L.: A restriction estimate using polynomial partitioning. J. Am. Math. Soc. 29(2), 371-413 (2016)

12. Xu, S., Yan, D.: A restriction theorem for oscillatory integral operator with certain polynomial phase. Front. Math. China 12(4), 967-980 (2017)

13. Carbery, A., Christ, M., Wright, J.: Multidimensional van der Corput and sublevel set estimates. J. Am. Math. Soc. 12(4), 981-1015 (1999)

14. Stein, E.M., Wainger, S.: Oscillatory integrals related to Carleson's theorem. Math. Res. Lett. 8(6), 789-800 (2001)

15. Shi, Z., Yan, D.: Sharp PP-boundedness of oscillatory integral operators with polynomial phases. Math. Z. 286(3-4), 1277-1302 (2017) 